\title{
Analysis of the dynamic characteristics of seawater intrusion using partial wavelet coherence: a case study at Nakaura Watergate, Japan
}

\author{
Edwin Muchebve $^{1}$ (D) Yoshiyuki Nakamura $^{1} \cdot$ Takayuki Suzuki $^{1} \cdot$ Hiroshi Kamiya $^{2}$
}

Published online: 31 October 2016

(c) The Author(s) 2016. This article is published with open access at Springerlink.com

\begin{abstract}
Lake Shinji and Lake Nakaumi are coupled brackish lakes on the Japan Sea coast of Japan. Seawater from the Japan Sea frequently intrudes into Lake Nakaumi through the bottom. Salinity flux at Nakaura Watergate was analysed. Effects of river discharge, tide level, and meteorological forcing variables makes the seawater intrusion non-linear. To account for the non-linear characteristics, partial wavelet coherence (PWC) was used to analyse the impact of river discharge, tide level, and meteorological forcing variables on salinity flux after controlling the effect of all other forcing variables. PWC results show that tide level has impact on salinity flux over short cycles (limited to less than 4 days) throughout the analysis period with an average time lag of about 2 days. This suggests that astronomical tides are the dominant factor in the salinity intrusion processes. The river discharge largely influences salinity flux over medium cycles (5-16 days) during the months of June and July with an average time lag of 0.3 days. Atmospheric pressure occasional affect tide level, indirectly driving salinity transport. The study findings provide guidance for factors affecting salinity intrusion processes and further research.
\end{abstract}

Keywords Continuous wavelet transform - Partial wavelet coherence $\cdot$ Salinity flux

Edwin Muchebve

muchebve-edwin-tg@ynu.jp

1 Graduate School of Urban Innovation, Yokohama National University, 79-5 Tokiwadai, Hodogaya-ku,

Yokohama 240-8501, Japan

2 Research Centre for Coastal Lagoon Environments, Shimane University, 1060 Nishikawatsucho, Matsue 690-8504, Japan

\section{Introduction}

Saline water intrusion process plays an important role in the ecosystems of estuaries and brackish water lakes. Salinity level and its fluctuation control dominant species in the pelagic and benthic ecosystems and thus alter structures of ecosystem. Saline water intrusion also affects water quality variations through formation of density stratification. Water exchange process is crucial for understanding of ecosystems and management of water quality in brackish water lakes. Water exchange between the Japan Sea and the couple brackish lake system of Lakes Shinji and Nakaumi, results in density stratification and hence the formation of oxygen-depleted water in the bottom layer during summer. The Sakai Channel is a major outlet of this coupled lake system, and seawater intrusion in this channel has seriously affected the habitat of aquatic species. During the summer, the vertical salinity gradient increases leading to anoxic bottom waters. Previous studies found that meteorologically induced sea surface variation (MISSV) and large periodic river discharge to be effective water exchange mechanism between the Japan Sea and Lakes Nakaumi and Shinji (Nakata et al. 2000). Tidal amplitude on the Japan Sea is small; hence, astronomical tides are not considered an effective water exchange mechanism. Meteorological forcing variables, e.g. atmospheric pressure and wind stress are also potential seawater intrusion mechanism. Salinity variation in Lakes Shinji and Nakaumi exhibits high correlations with the sea surface variation and river discharge (Nakata et al. 2000). However, the dynamic characteristic of salinity intrusion, and the effects of tide, river discharge and meteorological forcing variables on the salinity flux are unclear.

Wavelet analysis has been applied to the marine science, meteorological and hydrological fields so as to analyse 
non-stationary time series water quality data as well as meteorological data (Liu et al. 2014; Parmar and Bhardwaj 2013; Ng and Chan 2012; Zhang et al. 2009; Somoza et al. 2013; Ideião and Santos 2005; Sovi et al. 2012; Briciu 2014; Torrence and Compo 1998). Wavelet analysis has already been used to study salinity intrusion and river water quality parameters (Liu et al. 2014; Parmar and Bhardwaj 2013). However, most authors who applied wavelet analysis to study geophysical processes rely on univariate and bivariate wavelet analysis. Since salinity intrusion is influenced by more than one variable, the assessment of its association with any of the variables requires the consideration of its interaction with other variables. However, to the best of our knowledge, specifically about salinity intrusion dynamics, there is no previous work, which applied multivariate wavelet analysis. In order to assess the relationship between two-time series, it is important to remove the influence of other time series. Partial wavelet coherence (PWC) and partial phase-difference (PPD) enable the elimination of the influence of other time series, giving a clear estimation of the relationship between twotime series of interest.

Therefore, in order to understand the non-linear characteristics of salinity intrusion in Sakai Channel and quantify the relationships between river discharge, tide and meteorological forcing variables and salinity intrusion, this study applied the recent wavelet analysis techniquesPWC and PPD - to study the effects of river discharge, tide level and meteorological forcing variables on the salinity flux. Continuous Wavelet Transform (CWT) was used to analyse the period characteristics of tide level, river discharge, meteorological forcing variables and salinity flux.

\section{Materials and methods}

\subsection{Study area}

Lakes Shinji and Nakaumi form a coupled brackish lake system in the western part of Japan. Lake Shinji has an average depth of $4.5 \mathrm{~m}$, surface area of $80 \mathrm{~km}^{2}$ and volume of $0.366 \mathrm{~km}^{3}$. Lake Nakaumi has an average depth of $5.4 \mathrm{~m}$, surface area of $86.2 \mathrm{~km}^{2}$ and volume of $0.47 \mathrm{~km}^{3}$. The Ohashi River (7.0 km long) connects the two lakes and the Sakai Channel (7.5 km long) connects Lake Nakaumi to the Japan Sea. The Hii River at the west end of Lake Shinji supplies this coupled brackish lake system with most of its fresh water. Lake Shinji is a oligohaline lake with average salinity between 1 and 6 psu (Yamamuro et al. 1990). Lake Nakaumi has a strongly differentiated twolayer system, salinity of the surface water is 14-20 psu and that of the bottom layer is $25-30$ psu. Salinity in this lake system shows a seasonal variation, a marked horizontal gradient as well as vertical stratification mainly in Lake Nakaumi (Uye et al. 2000). Hence, these brackish lakes are stably stratified due to salinity (density) differences and density gradients have a large impact on water movement in this system (Okuda 2004; Mizoyama et al. 2011). Miho Bay is wide-open to east and north, and its hydrographic condition is almost oceanic. Sakai Channel is the sole outlet to the Japan Sea for the water flowing through Lake Nakaumi from Lake Shinji and small rivers and streams. The tidal range in Miho Bay is relatively small and rarely exceeds $0.4 \mathrm{~m}$. However, tidal currents are quite strong, sometimes reaching about $0.5 \mathrm{~m} / \mathrm{s}$ (Harada 1968).

The high saline water, flowing through Sakai Channel, entering into Lake Nakaumi from Miho Bay is not diluted by the surface water and the salinity of the bottom water of Lake Nakaumi is only slightly lower than that of Miho Bay (Harada 1968; Nakata et al. 2000). Seawater entering Lake Nakaumi also travels along the bottom upstream the mouth of Ohashi River. Brackish water from Lake Shinji flows into Lake Nakaumi through Ohashi River forming the surface layer. Bottom layer with high salinity in Lake Nakaumi is occasionally driven by the wind-induced internal waves and tides towards the lake coast and upstream into Ohashi River and Lake Shinji (Ishitobi et al. 1999, 1989). The effect of wind stress on internal oscillation is greater than that of astronomical tides. However, at Nakaura Watergate, due to the orientation of the channel, the predominantly westerly winds have less effect (Fujii and Okuda 2004).

The average water transport direction in Ohashi River is eastward, from Lake Shinji to Lake Nakaumi. This eastward water transport approximately equals the freshwater inflow into Lake Shinji, mainly from the Hii River (Nakata et al. 2000). The westward water transport, mainly driven by MISSV, occurred often with the time scale over a few days to a week, contributing to the exchange of water between the two lakes (Nakata et al. 2000). The salinity in Ohashi River, between Lakes Shinji and Nakaumi, vary acutely as a result of water moving back and forth with tides, freshwater input and other physical factors (Uye et al. 2000; Ishitobi et al. 1989, 1999).

\subsection{Observations}

Salinity and current velocity data used in the study was collected at Nakaura Watergate monitoring station (insert, Fig. 1). The gate was originally constructed to close the channel to stop saline water intrusion, in order to make the lake fresh water lake for irrigational purposes, However, the planning has recently stopped and the gate was removed. Nakaura Watergate (width $414 \mathrm{~m}$, depth $6.8 \mathrm{~m}$ ) had five floodgates on the east and on the west (each $32 \mathrm{~m}$ wide), and three floodgates at the centre. On the western 


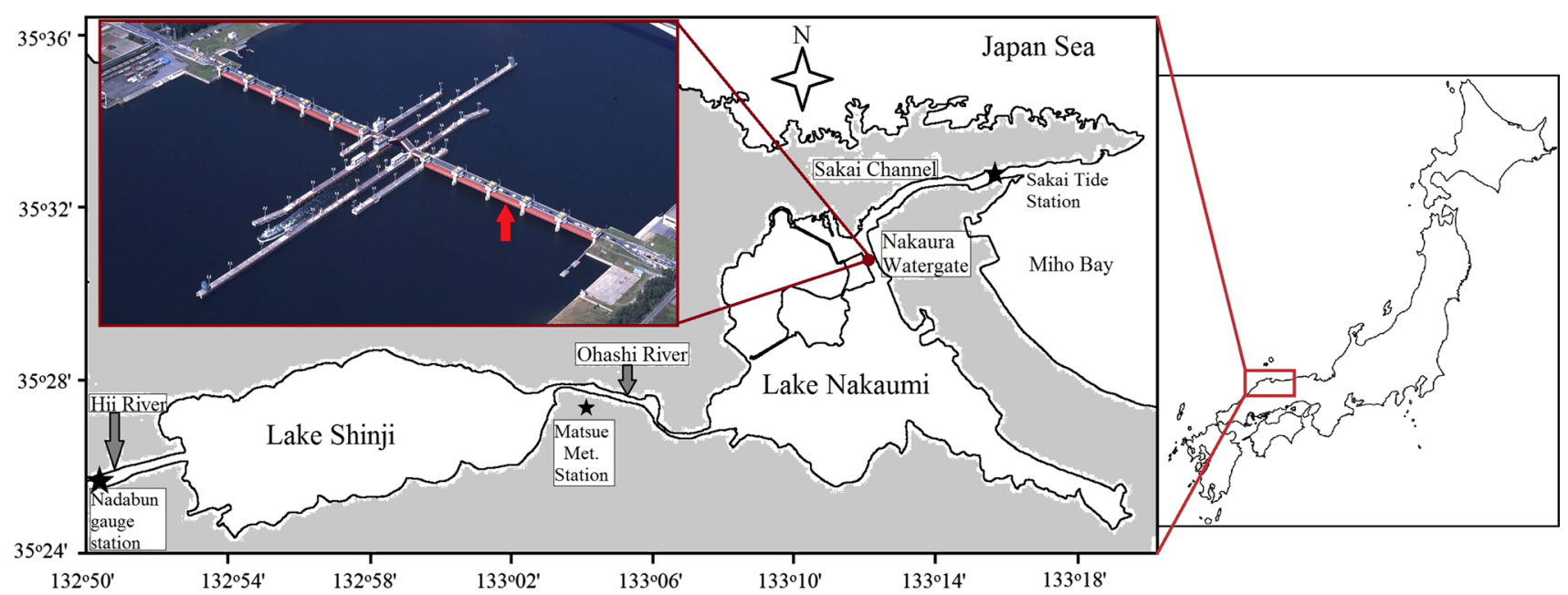

Fig. 1 Location of Lakes Shinji and Nakaumi, and Nakaura Watergate monitoring station (insert). Red arrow indicates the 3rd eastern side floodgate, the location of sampling and measurement equipment. Also shown are meteorological and hydrological monitoring stations

pile of the 3rd east side floodgate (indicated by red arrow, Fig. 1), submerged water pumps were installed for water sampling at 1,2, 4 and $6 \mathrm{~m}$ from the bottom and the water pumped to acrylic boxes in the floodgate administration building, where water temperature, electrical conductivity and dissolved oxygen were measured every $30 \mathrm{~min}$ using custom-made sensors (Alec Electronics Co., Ltd.). Salinity was then calculated from electrical conductivity. Acoustic Doppler Profiler (Nortek ADP) was also installed in the centre of the 3rd floodgate on the eastern side of the Nakaura Watergate (indicated by red arrow, Fig. 1), near the bottom. Current velocity measurements were taken every half hour at depth intervals of $0.5 \mathrm{~m}$, from 1 to $6 \mathrm{~m}$ above the bottom. Along-channel current vector component is used in this study. Positive along-channel velocities were ebb directed (northward flow), whilst negative values were associated with southward (flood) flow. The orientation of Nakaura Watergate was in the east-west direction and the direction of flow at this location is almost southnorth, that is, ebb tidal current is northwards and flood tidal current southwards.

Continuous measurements of salinity over a period of six years (February 1998 to March 2004) is available, although there are periods with missing data. Salinity, water temperature, current velocity and water level data during the summer of 2002 (June to September 2002) was used to calculate salinity flux per unit width in this study. High saline water intrusion during the summer season is important for water quality deterioration; as it directly cause salinity stratification and enhance oxygen depletion in the bottom layer (Nakata et al. 2000). Also this the period has least missing data. The salinity, water temperature, current velocity and water level data was averaged to one-hour intervals to match the intervals of the meteorological data used in the analysis. One-hour interval meteorological data (atmospheric pressure, wind speed and direction) was collected at Matsue Meteorological Station (available on Japan Meteorological Agency website, http:// www.jma.go.jp/jma/index.html). The wind was treated as a mathematical vector, and the mathematical convention for the direction was used, i.e., wind direction was converted from "meteorological direction" to "math direction". The wind vector was resolved into its $x$ and $y$ components. Wind from the west positive $W_{x}$, and from the south a positive $W_{y}$. Tidal data used in the study was recorded at Sakai Tide Station maintained by Japan Meteorological Agency (http://jdoss1.jodc.go.jp/cgi-bin/1997/tide_data? MA53). River discharge was recorded in Hii River at Nadabun gauging station (available on Japan's water information system website, http://www1.river.go.jp/).

\subsection{Methodology}

\subsubsection{Salinity transport}

Instantaneous advective salt transport $\left(M_{s}, \mathrm{kgm}^{-1} \mathrm{~s}^{-1}\right)$ per unit width of a section, normal to the longitudinal flow of the channel, is given by the following expression (Vaz et al. 2012; Moser et al. 2005):

$M_{s}=\int_{0}^{h} \rho V S d z=\overline{\rho V S} \cdot h$

where $\rho$ is the density, $V$ is the longitudinal velocity component, and $S$ is the longitudinal salinity. The upper bar denotes averaging over the total depth of the water column, $h$. 
The density of water was calculated using the following approximate density formula neglecting pressure (shallow lake) (Klinger n.d.):

$\rho=999.83+0.808 S-0.0708(1+0.068 T) T$

$$
-0.003(1-0.012 T)(35-S) T
$$

where $T$ is temperature in ${ }^{\circ} \mathrm{C}$, and $S$ is salinity in PSU.

\subsubsection{Continuous wavelet transform (CWT)}

CWT decomposes a time series into a time-frequency space and determines both the dominant modes of variability and their variation with time (Torrence and Compo 1998). The wavelet is applied as a bandpass filter to the time series, stretching it in time by varying its scale(s) and normalizing it to have unit energy (Grinsted et al. 2004; Zhang et al. 2009).

A wavelet $\psi(t)$ is a function that oscillates around the $t$ axis and looses strength as it moves away from the centre, behaving like a small wave (Torrence and Compo 1998). Beginning with a mother wavelet $\psi$, a family of "daughter wavelet", $\psi(\tau, s)$ is computed by scaling and translating $\psi$ :

$\psi_{\tau, s}(t)=\frac{1}{\sqrt{|s|}} \psi\left(\frac{t-\tau}{s}\right) ; s, \tau \in \mathbb{R} ; s \neq 0$

where $\psi(t)$ : mother wavelet, $\psi_{\tau, s}(t)$ : daughter wavelet, $t$ : a non-dimensional "time" parameter, $s$ : a scaling or dilation factor that controls the width of the wavelet, $\tau$ : a translation parameter controlling the location of the wavelet.

This study used the Morlet wavelet, which consists of a plane wave modulated by a Gaussian or in other words, a complex exponential function multiplied by a Gaussian window. Hence represents the best compromise between frequency and time localization. A complex wavelet is essential for this study, as it yields a complex transform, with information on both the amplitude and phase, crucial to study the synchronization of oscillations between different time-series (Sousa 2014; Aguiar-Conraria and Soares 2014). Morlet wavelet is defined as:

$\psi_{0}(t)=\pi^{-1 / 4} e^{i \omega_{0} t} e^{-t^{2} / 2}$

where $\psi_{0}(\mathrm{t})$ : the Morlet wavelet, $\omega_{0}$ : wavenumber, gives the number of oscillations within the wavelet itself

CWT of a time series $x(t) \in L^{2}(\mathbb{R})$, with respect to the wavelet $\psi$ is a function of two variables, $W_{x ; \psi}(\tau, s)$ :

$W_{x ; \psi}(\tau, s)=\int_{-\infty}^{\infty} x(t) \frac{1}{\sqrt{|s|}} \psi^{*}\left(\frac{t-\tau}{s}\right) d t$

where $W_{x ; \psi}(\tau, s)$ : CWT of a time series $x(t), L^{2}(\mathbb{R})$ : denotes the set of square integrable functions, i.e. the set of functions defined on the real line and satisfying $\int_{-\infty}^{\infty}|x(t)|^{2} d t<\infty, \psi^{*}:$ complex conjugation of $\psi$

\subsubsection{Partial wavelet coherence}

CWT is increasingly being used in the analysis of marine sciences time series data. However, most of the CWT analysis has been limited to univariate and bivariate analysis, i.e., the wavelet power spectrum, the wavelet coherency and the wavelet phase-difference (Aguiar-Conraria and Soares 2014). Wavelet analysis tools have already been extended to allow for multivariate analyses $(\mathrm{Ng}$ and Chan 2012; Aguiar-Conraria and Soares 2014). PWC and PPD are the examples of recent wavelet analysis techniques. The PWC technique is similar to partial correlation and it identifies the resulting wavelet coherence between two time series after eliminating the influence of their common dependence ( $\mathrm{Ng}$ and Chan 2012). The applicability of PWC to geophysics was demonstrated during the study of the "stand-alone", relationship between the "ratio of number of typhoons to number of tropical cyclones" and "large-scale atmospheric factors" after removing the effect of El Nino-Southern Oscillation (ENSO) ( $\mathrm{Ng}$ and Chan 2012).

The squared multiple wavelet coherence $\left(R_{1(23 \ldots p)}^{2}\right)$ between the series $x_{1}$ and all the other series $x_{2}, \ldots, x_{p}$ is given by the formula

$R_{1(23 \ldots p)}^{2}=1-\frac{C^{d}}{C_{11}^{d}}$

where $C$ : denote the $p \times p$ matrix of all the complex wavelet coherencies $\varrho_{i j}$, i.e. $\mathrm{C}=\left(\varrho_{i j}\right)_{i, j=1}^{p}, C^{d}=\operatorname{det} C$

The complex partial wavelet coherence $\left(\varrho_{1 j . q_{j}}\right)$ of $x_{1}$ and $x_{j}(2 \leq j \leq p)$ allowing for all the other series is given by

$\varrho_{1 j \cdot q_{j}}=-\frac{C_{j 1}^{d}}{\sqrt{C_{11}^{d}} \sqrt{C_{j j}^{d}}}$

where $q_{j}$ is the set of all the indexes from 2 to $p$ with the exception of $j$, i.e. $q_{j}=\{2, \ldots, p\} \backslash\{j\}$.

The partial wavelet coherence $\left(R_{1 j . q_{j}}\right)$ of $x_{1}$ and $x_{\mathrm{j}}$ allowing for all the other series, is defined as the absolute value of Eq. 7, i.e.

$R_{1 j . q_{j}}=\frac{\left|C_{j 1}^{d}\right|}{\sqrt{C_{11}^{d} C_{j j}^{d}}}$

and the squared partial wavelet coherence of $x_{1}$ and $x_{\mathrm{j}}$ allowing for all the other series, is simply the square of $R_{1 j . q_{j}}$.

The partial phase-delay (phase-difference) of $x_{1}$ and $x_{\mathrm{j}}$, given all the other series is defined as the angle of $\varrho_{1 j . q_{j}}$. A complex wavelet function contains information about both the amplitude and the phase, making makes it suitable to capture oscillatory behaviour. Complex partial wavelet 
coherence, $\varrho_{1 j \cdot q_{j}}$ considered can be separated into its real part, $\Re\left(\varrho_{1 j \cdot q_{j}}\right)$ and imaginary part, $\mathfrak{J}\left(\varrho_{1 j \cdot q_{j}}\right)$ or in its amplitude, $\left|\varrho_{1 j \cdot q_{j}}\right|$, and phase angle $\phi_{1 j \cdot q_{j}}$. The phase-difference, $\phi_{1 j . q_{j}}$ is given as follows:

$\phi_{1 j \cdot q_{j}}=\operatorname{Arctan}\left(\frac{\mathfrak{I}\left(\varrho_{1 j \cdot q_{j}}\right)}{\Re\left(\varrho_{1 j \cdot q_{j}}\right)}\right)$

A phase-difference of zero indicates that the time series move together at the specified time-frequency; if $\phi_{x_{1} x_{j}} \in(0,90)$, the series move in phase and the time series of $x_{1}$ leads $x_{\mathrm{j}}$; if $\phi_{x_{1} x_{j}} \in(-90,0)$, then $x_{\mathrm{j}}$ leads $x_{1}$. A phasedifference of 180 (or -180 ) indicates an antiphase relationship; if $\phi_{x_{1} x_{j}} \in(90,180)$, then $x_{1}$ leads $x_{\mathrm{j}}$; if $\phi_{x_{1} x_{j}} \in(-180,-90)$, then $x_{\mathrm{j}}$ leads $x_{1}$. Phase difference can be converted into instantaneous time-lag between two-time series by dividing the phase-difference, $\phi_{1 j . q_{j}}$ by the angular frequency corresponding to the scale $\mathrm{s}, \omega(s)$

\subsubsection{Significance tests}

The assessment of the statistical significance of the wavelet power spectrum and the wavelet coherence results is important. Monte Carlo methods were used to assess the statistical significance levels and confidence intervals against red noise backgrounds.

\section{Results and discussion}

\subsection{Analysis of period characteristics using continuous wavelet transform}

The analysis variables and their wavelet power spectrum are shown in Figs. 2, 3, 4, 5, and 6. The intensity of the wavelet power shows the time series variance for each moment of time and each frequency of oscillations. Figure $2 \mathrm{a}$ show the salinity flux per unit width at Nakaura Watergate. Positive values indicate flux towards the Japan Sea and negative towards Lake Nakaumi. The CWT coefficient chart for salinity flux (Fig. 2b) has stable period characteristics, with high power oscillations in the 0.5-day and 1-day period bands. Both the red colour and the black contour indicates that cycles are strong and statistically significant at $95 \%$ confidence level (hereinafter statistically significant). Also observed is a relatively strong, though not statistically significant, 2-4-day period cycle that occurs in first week of September. This behaviour coincides with the statistically significant oscillations for tide level shown in Fig. 3. From mid-June to mid-July and end of August,

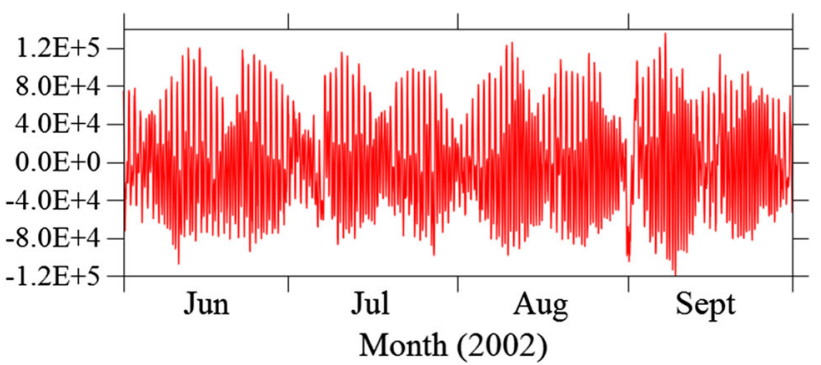

(a) Salinity Flux $\left(\mathrm{kgm}^{-1} \mathrm{~s}^{-1}\right)$

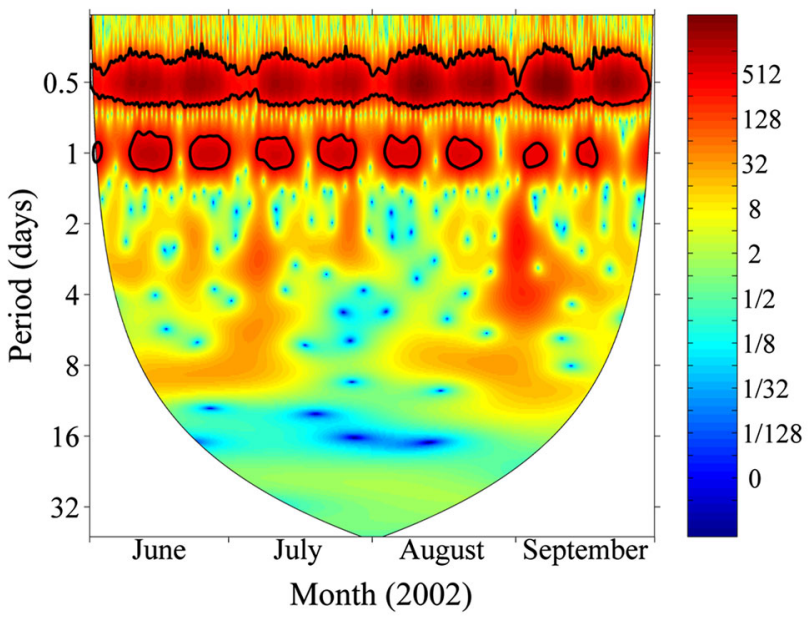

(b) Wavelet Power Spectrum for Salinity Flux

Fig. 2 The time series of salinity flux and its wavelet power spectrum. The black contour designates $95 \%$ confidence level, using red noise as background spectrum. White regions on either end indicate the "cone of influence" where edge effects become important

there was change in salinity flux and this possess an 8-day period.

Figure 3 shows time series plot and CWT coefficient chart for the tide level showing continuous and fluctuating low and medium power oscillations appearing around 0.5 and 1 day periods, respectively. These oscillations appear twice a month, which means spring-neap tidal variations. Even for the power with half day or one day variation, phenomenon with longer periodicity and its variations can be visible. The tide level has statistically significant periods of half a day and one day. This implies considerable power spreads throughout the semi-diurnal and diurnal bands in summer. Figure 3 also indicates high power oscillations when the tide level increased and dropped from the end of August to early September. This increase and drop maybe a result of the influence of meteorological forcing variables such as wind or atmospheric pressure. Though not statistically significant, time series plot and CWT coefficient chart indicate impulsive increases in tide level appearing several times in mid-June, early July, and the beginning of September. Such changes seem to be corresponding to sudden drop in atmospheric pressure (Fig. 5). The latter 


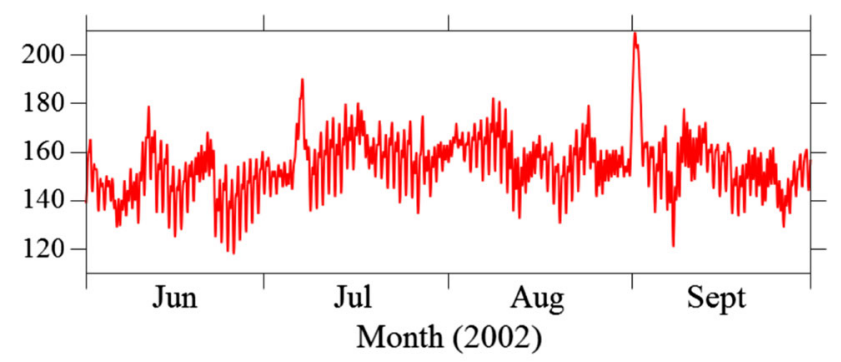

(a) Tide Level (cm)

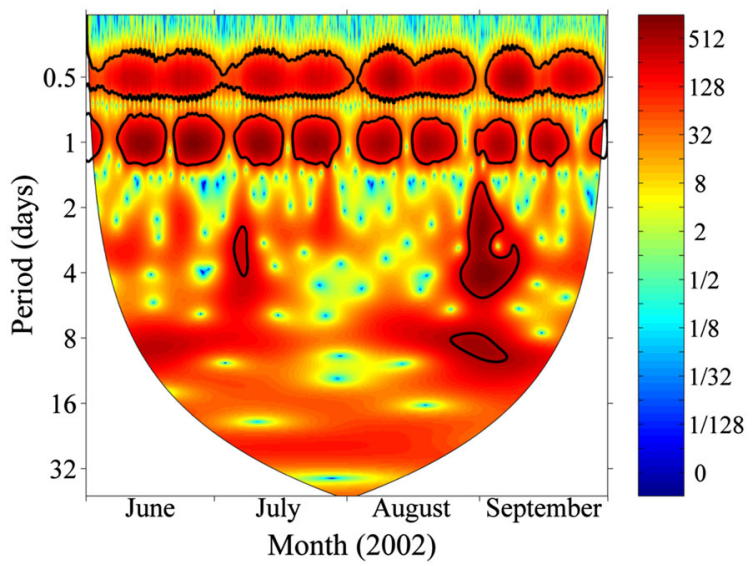

(b) Wavelet Power Spectrum for Observed Tides

Fig. 3 Tide Level-time-series plot and time-series wavelet power spectrum

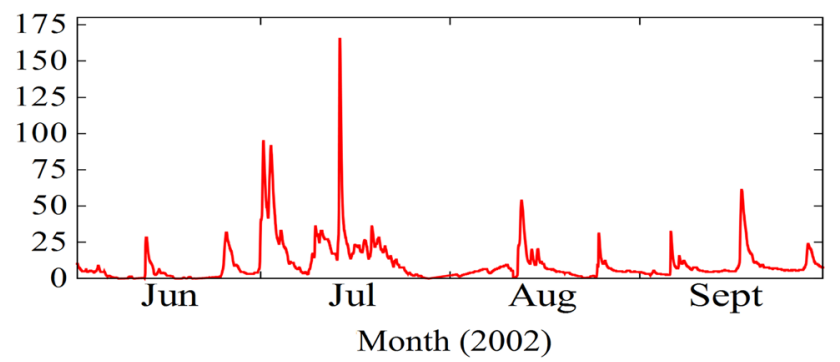

(a) River Discharge $\left(\mathrm{m}^{3} / \mathrm{s}\right)$

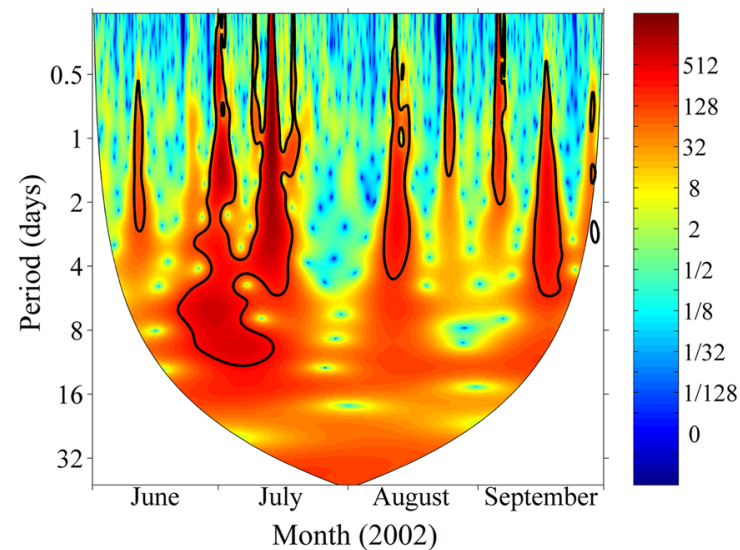

(b) Wavelet Power Spectrum for River discharge

Fig. 4 River discharge-time-series plot and time-series wavelet power spectrum

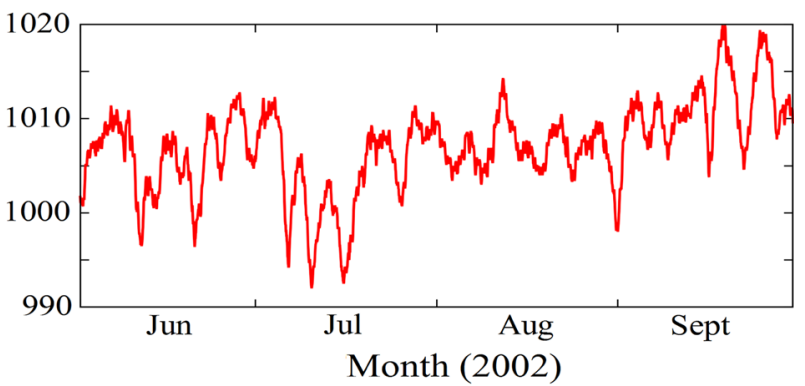

(a) Atmospheric Pressure $(\mathrm{hPa})$

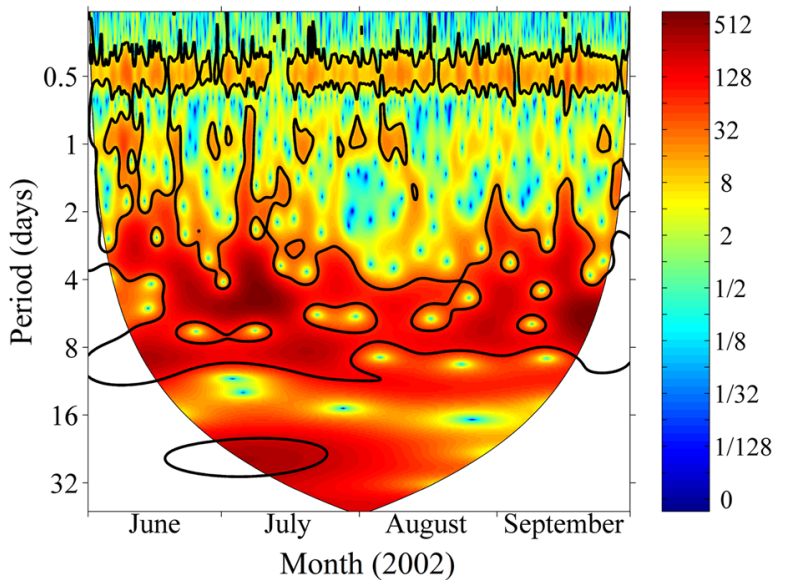

(b) Wavelet Power Spectrum for Atmospheric pressure

Fig. 5 Atmospheric pressure-time-series plot and time-series wavelet power spectrum

two events seem to be corresponding to increased salinity transport, however, such events which occur at random with shorter duration are not well reproduced by the wavelet analysis. This indicates a possible weakness of the wavelet analysis, a situation whereby intrusion processes are not always fully described.

Figure 4 show time series plot and CWT coefficient chart for the river discharge, which shows that the river discharge wavelet coefficients vary from the highest to the lowest indicating a highly irregular river discharge. The chart also shows distinct character that has long vertical peaks like a rain drop which indicate that the period of oscillation varies from high to low almost instantaneously. Each peak of energy corresponds to high river discharge and its slower decrease in flow rate. From the time series plot and CWT coefficient chart, it is evident that highest river flow rate occurs between mid-June and mid-July. A strong and statistically significant 4-day period cycle is consistent for about 2 weeks in July. There are also discontinuous and regular relatively high river discharges in August and September. The irregularity of Hii River discharge may be attributed to non-stationary rainfall and water releases upstream. The increased river discharge in July contributed to an increase sea surface elevation in Sakai Channel (Fig. 3). 


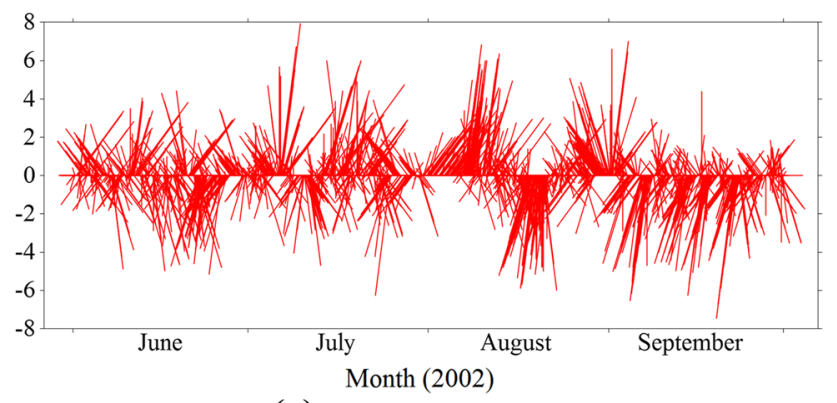

(a) Wind Vector $(\mathrm{m} / \mathrm{s})$

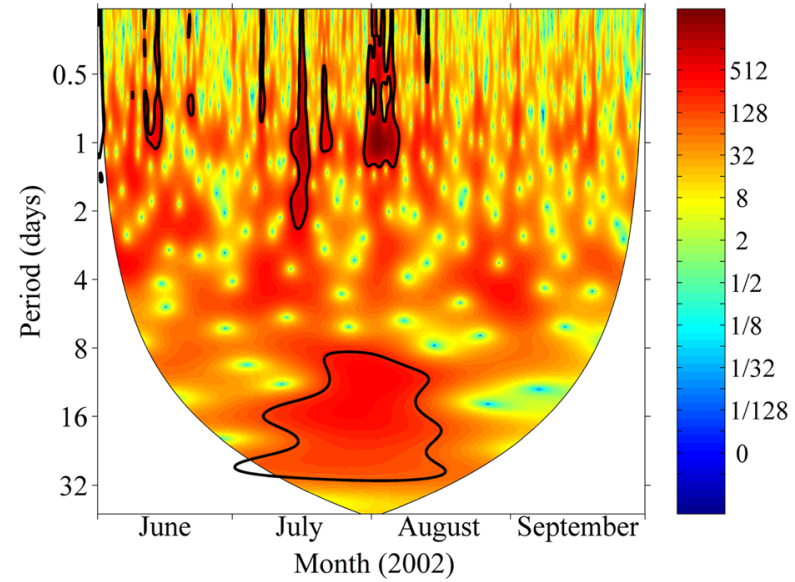

(b) Wavelet Power Spectrum for E-W Wind vector

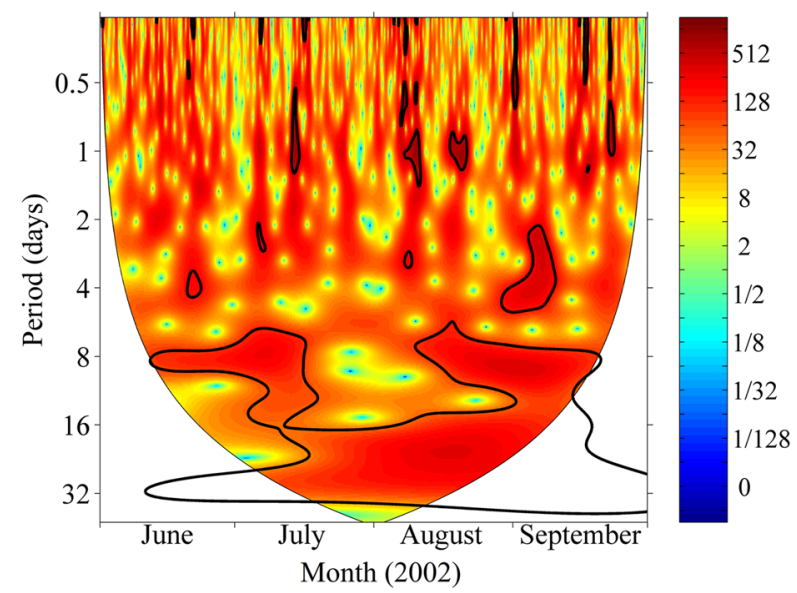

(c) Wavelet Power Spectrum for N-S Wind vector

Fig. 6 Wind vector-time-series plot and time-series wavelet power spectrum

The CWT coefficient chart for the atmospheric pressure (Fig. 5b) shows continuous statistically significant medium power 4-8-day period cycles throughout the analysis period. This strongly indicates the effect of mesoscale atmospheric physics in the mid-latitude of the northern hemisphere. The average life span of a low pressure or high pressure air mass is about seven days. Continuous medium power oscillations are observed in the 0.5-day band. There are also discontinuous and irregular medium power oscillations possessing a 1-day period. The time series plot indicates a statistically significant decrease in pressure in mid-June, mid-July and beginning of September. This pressure drop resulted in increased sea surface elevation as shown in Fig. 3 and increased salinity intrusion into Lake Nakaumi as shown in Fig. 2.

The CWT coefficient charts for wind vectors (Fig. 6) show that stable statistically significant high power oscillations appear at longer periods, 8-32-day period band. The east-west wind component shows irregular fluctuating medium power in the 1-day period band in June, July and August. Strong and statistically significant 8, 16 and 32-day period cycles are observed. The east-west wind component also shows a 4-day medium power cycle occurring early June, mid-July and end of August (Fig. 6b). The northsouth component also shows occurrences of medium power oscillations with a 4-day period during mid-June and early of September (Fig. 6c). Medium power oscillations are also observed in the 8-day period (mid-June to mid-July, and mid-August to mid-September) and in the 32-day band. Statistically significant high power wind variation occurred in August cantered in the 24-day period cycle. Generally, the strong wind variation was observed mainly in the eastwest component during the month of July. From mid-August to early September, the north-south component dominated. From early August to mid-August the variation in both components was strong and statistically significant.

\subsection{Analysis of dynamic characteristics using partial wavelet coherence}

Partial coherence and partial phase-difference, described above, are used to estimate the interdependence, in the time-frequency domain, between two series after eliminating the influence of other series. Figures 7, 9, 10 and 11 shows the interdependence, in the time-frequency domain, between salinity flux and each of the forcing variables, after eliminating the effect of other variables. The partial phase difference is the average partial phase difference for each frequency band. It is possible to calculate the phase difference for each frequency. In this study, three frequency bands (1/2-4 day, 5-16 day, and 17-32 day) were chosen because the oscillations of all the variables were almost divided among these frequency bands. Table 1 shows mean phase difference and mean lag time with forcing factors leading for both in-phase and out-of-phase situations. Figure 8 shows the general ratio of occurrences when tides and river discharge were in-phase or out-ofphase, leading or lagging salinity transport.

\subsubsection{Correlation between tide level and salinity flux}

Figure 7 shows extensive statistically significant power sections at the 5\% level, indicating the existence of 


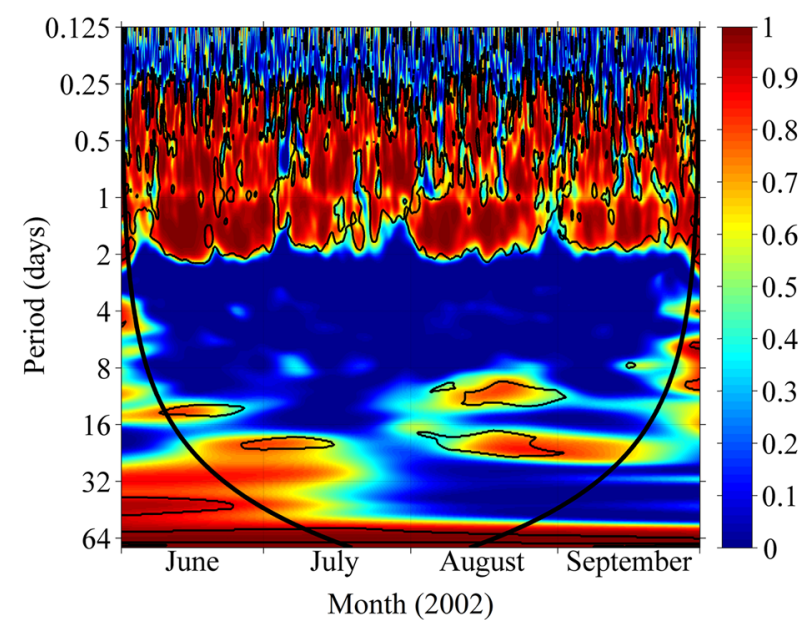

(a) Partial Wavelet Coherency (tides,sstrans;(river,atm,wx,wy))

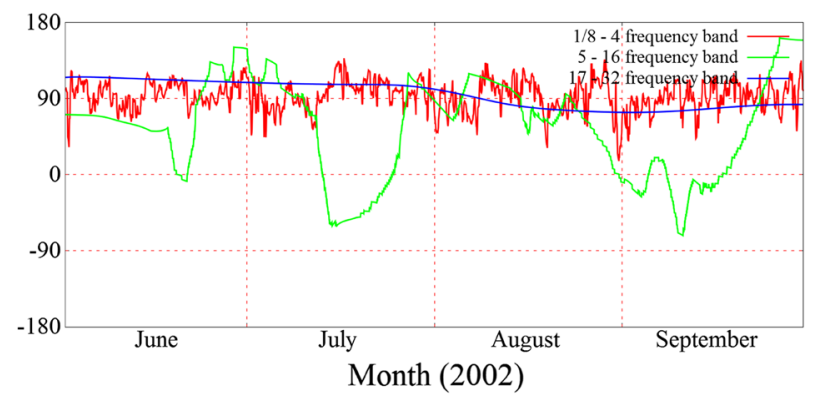

(b) Partial Phase Difference - tides,sstrans;(river,atm,wx,wy)

Fig. 7 Observed tides versus salinity flux partial wavelet coherence and partial phase-differences. Thick black line indicates the cone of influence that delimits the region not influenced by edge effects

correlation between observed tides and salinity transport. The centre of power sections focuses on periods $12 \mathrm{~h}$ and 1-day. Figure $7 \mathrm{~b}$ shows that relationship between tides and salinity flux is typically between 0 and $180^{\circ}$, indicating that tides are leading. Since an increase rise in tide level leads to an increase in salinity flux into Lake Nakaumi (denoted as negative flux in this study), the phase relationship

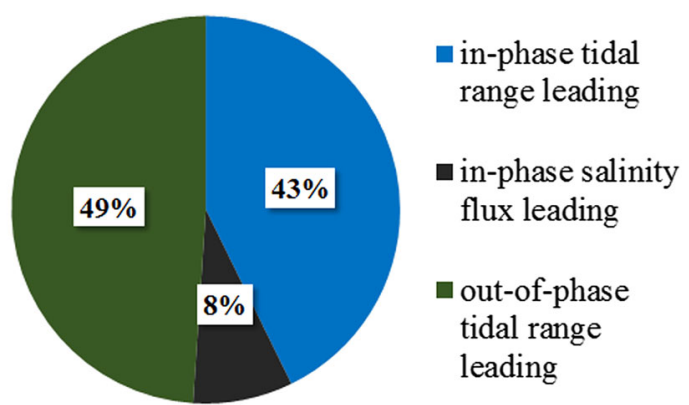

(a) Tide Level vs. Salinity Flux

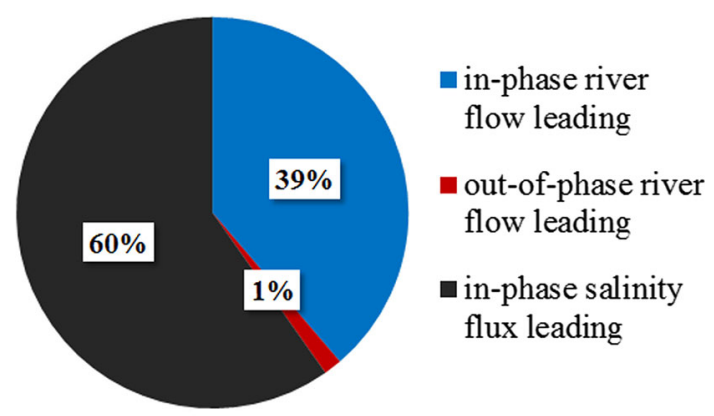

(b) River Discharge vs. Salinity Flux

Fig. 8 Phase relationships-river discharge and tidal range versus salinity flux

between tide level and salinity flux is opposite the normal notation. Therefore, phase difference between 90 and $180^{\circ}$ indicate positive correlation (in-phase) between tide level and salinity flux with tide level leading. Phase difference between 0 and $90^{\circ}$, indicate negative correlation (out-ofphase) between tide level and salinity flux with tide level leading. Most of the time tide level has a positive impact on salinity flux, over the periods $12 \mathrm{~h}$ and 1-day. This implies that short-term salinity transport is highly influenced by tides. Tide level also has statistically significant positive correlation with salinity flux in the 15-day period band in
Table 1 Mean phase difference and time lags between the forcing variables and the salinity flux

\begin{tabular}{lcc}
\hline Forcing variable & Mean phase difference & Mean time lag \\
\hline In-phase & & \\
Tidal range (tides) & 109.3 & 4.0 \\
River discharge (river) & 16.6 & 0.3 \\
Atmospheric pressure (atm) & 28.0 & 0.1 \\
Wind vector (east-west) (wx) & 52.4 & 2.0 \\
Wind vector (north-south) (wy) & 43.2 & 1.5 \\
Out-of-phase & & \\
Tidal range (tides) & 71.8 & 2.3 \\
River discharge (river) & 95.5 & 1.4 \\
Atmospheric pressure (atm) & 90.4 & 0.3 \\
Wind vector (east-west) (wx) & 92.9 & 0.3 \\
Wind vector (north-south) (wy) & 100.7 & 0.3 \\
\hline
\end{tabular}


June and August, this could be the result of spring tides. In August and September, there positive relationship between salinity flux and tides centred around 20-day period. Almost half the time, salinity flux and tide level are inphase with salinity flux leading, resulting in seawater intrusion into Lake Nakaumi (Fig. 8a). The high tide takes an average of 4 days to have net salinity flux towards Lake Nakaumi (Table 1). Table 1 also shows that tide level is usually out-of-phase and earlier than salinity flux by an average of 2.3 days. This imply that after the tides have subsided it takes an average of 2.3 days to have net salinity flux towards Miho Bay. Though the tidal range in the Japan Sea, tidal currents have proven to be important in the transportation of salinity in and out of Lake Nakaumi.

\subsubsection{Correlation between river discharge and salinity flux}

PWC and PPD between river discharge and salinity flux, after controlling for other forcing variables, show statistically significant in-phase relationship in the 5-16-day period from early June to mid-July. This affirms the existence of the correlation between river discharge and salinity flux. Statistically significant high power coherence observed in June and July coincides with increased river discharge. The inphase relationship means as the river discharge increases salinity being transported also increases. As shown in Fig. 9b, the phase lags in the 1-4-day band are mainly inphase between -90 and $90^{\circ}$ and exhibiting very unstable lead-lag relationships between river discharge and salinity flux. The river discharge leads salinity flux and in phase in the 8-16-day period band, around the beginning of September. This can be the effect of increased river flows due to typhoon events. The increased river flow rate enhances estuarine circulation (increased salinity flux along the bottom layer). For long-term period band (17-32), there is no statistically significant in-phase relationship, though beginning mid-August, river discharge was leading salinity transport. Figure $8 \mathrm{~b}$ and Table 1 indicate that in about $39 \%$ of the time river discharge is in-phase and leads salinity transport by about 0.3 days. Most of the time salinity flux is ahead of river discharge due to other transport mechanisms.

The discharge rate of Hii River, may flush salinity and as well as increasing in bottom salinity of Lake Nakaumi (Nakata et al. 2000). The time scale of this change may be residence time (beyond the scope of this study). A numerical study may be needed to understand the residence time in relation to the phase lag from wavelet analysis.

\subsubsection{Correlation between atmospheric pressure and salinity flux}

The statistically significant relationship between atmospheric pressure and salinity flux exists, though

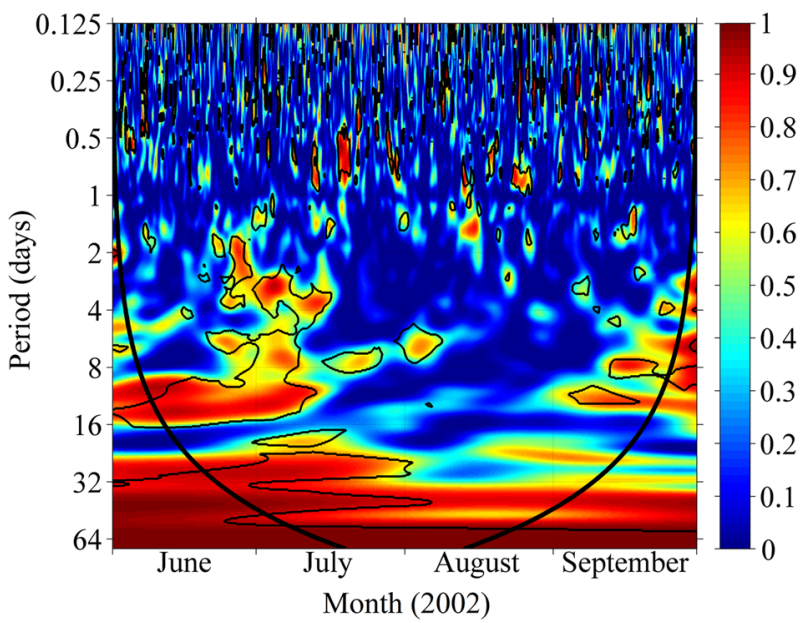

(a) Partial Wavelet Coherency (river,sstrans;(tides,atm,wx,wy))

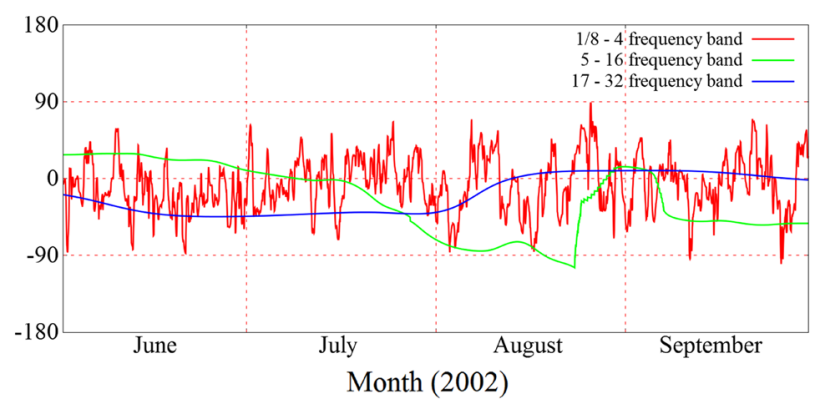

(b) Partial Phase Difference - river,sstrans;(tides,atm,wx,wy)

Fig. 9 River discharge versus salinity flux partial wavelet coherence and partial phase-differences

discontinuous and irregular, indicating the existence of correlation between atmosheric pressure and salinity flux. Figure $10 \mathrm{~b}$ shows that the phase difference is typically between -90 and $90^{\circ}$ and exhibiting very unstable lead-lag relationships between atmospheric pressure and salinity flux. Atmospheric pressure leads and in-phase with salinity flux in the 0.5-4-day period band. It's influence on the flux of salinity is not stable, implying it is short-lived and weak influence on salinity flux.

\subsubsection{Correlation between wind vectors and salinity flux}

Figure 11 shows that the phase lags in the 3-h-4-day band are mainly in-phase between -90 and $90^{\circ}$ and exhibiting very unstable lead-lag relationships between wind vectors and salinity flux. However, in this period band salinity flux is mostly leading wind vectors. The east-west wind component $(w x)$ and salinity flux are mainly in-phase with wind vector leading in the periods between 5 and 32-day (Fig. 11a). The statistically significant correlation between the east-west wind component and salinity flux exist mainly in the 8-16-day period band, appearing consistently in August (Fig. 11a, b). This indicates that relatively strong westerly winds have the potential to drive salinity out Lake 


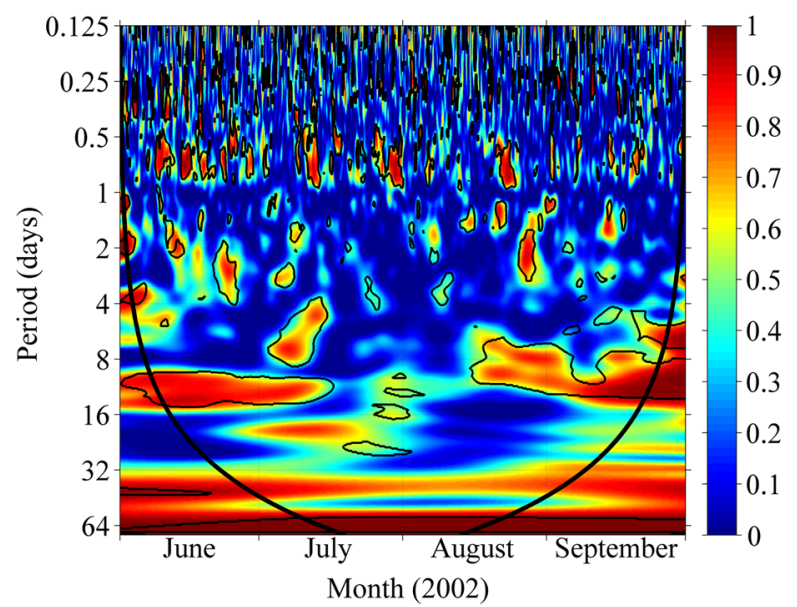

(a) Partial Wavelet Coherency (atm,sstrans;(river,tides,wx,wy))

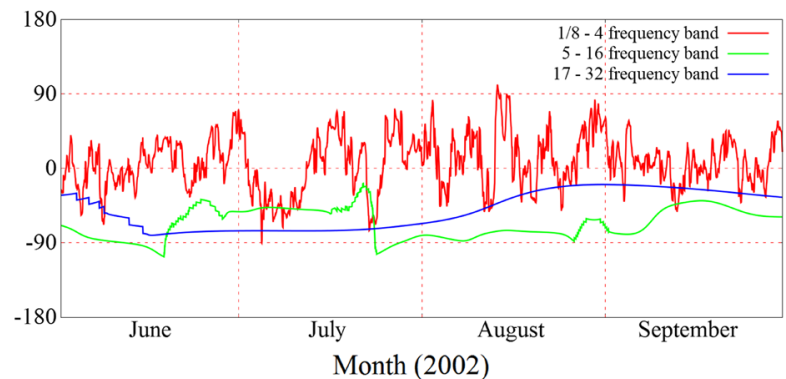

(b) Partial Phase Difference - atm,sstrans;(river,tides,wx,wy)

Fig. 10 Atmospheric pressure versus salinity flux partial wavelet coherence and partial phase-differences

Nakaumi. The north-south wind component (wy) has statistically significant power sections in the 5 and 32-day period band with antiphase relationship with and leading salinity flux. This means the southerly wind (positive wind speed) drives salinity into Lake Nakaumi (negative salinity flux), and northerly wind (negative wind speed) flush salinity out of Lake Nakaumi, hence antiphase relationship (Fig. $11 \mathrm{c}, \mathrm{d}$ ). Wind seems to promote mixing and oscillation within Lake Nakaumi. From Fig. 11 it is clear the east-west wind component has little effect on salinity flux supporting observations by previous studies (Fujii and Okuda 2004). Wind vectors usually leads salinity flux by an average of 2 days and 1.5 days for east-west and northsouth wind component, respectively (Table 1).

\subsection{Implications of the study results}

Astronomical tides being regular were the dominant parameter affecting sea surface elevation. It is also evident from Figs. 3 and 5 that sea surface elevation in Sakai Channel is affected by atmospheric pressure. The pressure drop in mid-June, mid-July and beginning of September resulted in an unusual increase in sea surface elevation. Salinity transport also responded to this change. River

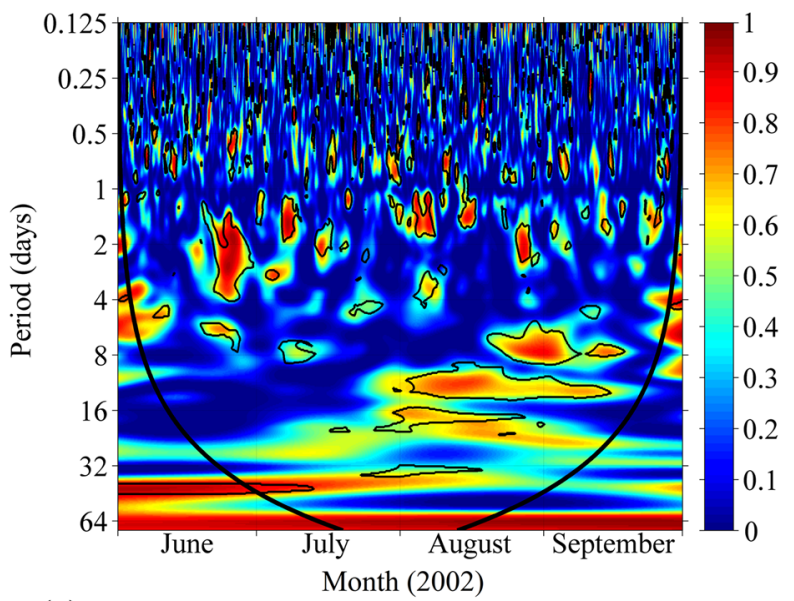

(a) Partial Wavelet Coherency (wx,sstrans;(river,tides,atm,wy))

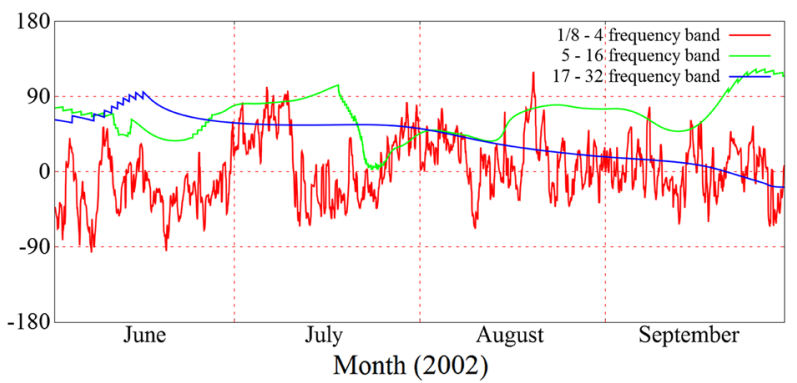

(b) Partial Phase Difference - wx,sstrans;(river,tides,atm,wy)

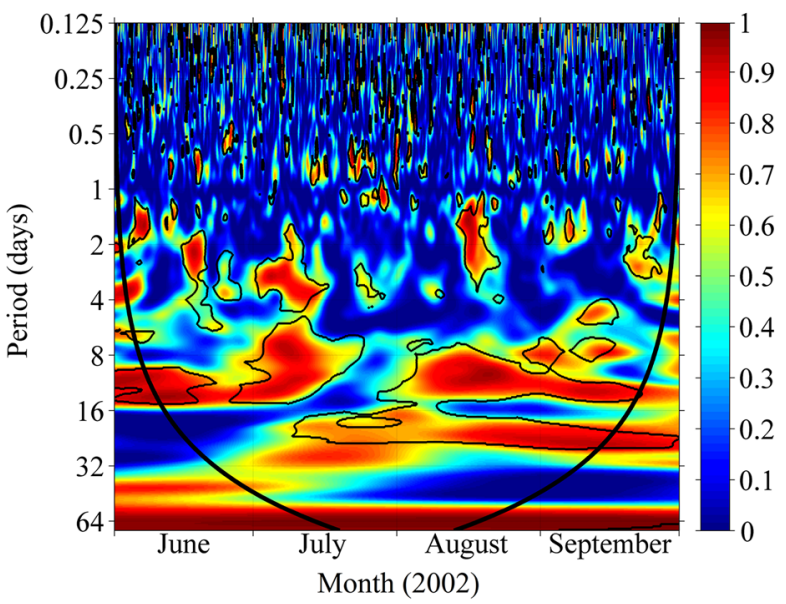

(c) Partial Wavelet Coherency (wy,sstrans; (river,tides, atm,wx))

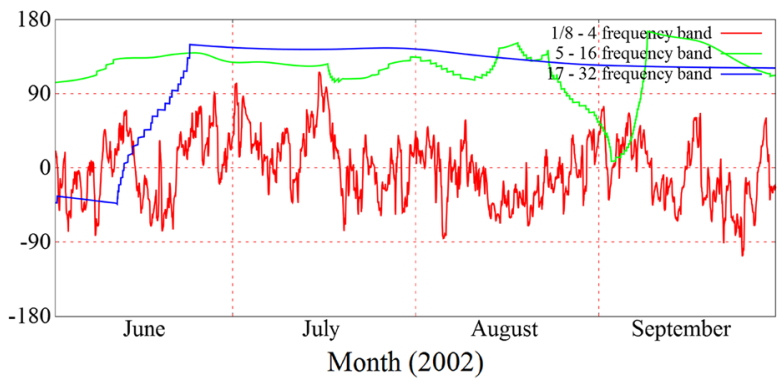

(d) Partial Phase Difference - wy,sstrans;(river,tides,atm,wx)

Fig. 11 Wind vectors versus salinity flux partial wavelet coherence and partial phase-differences 
discharge occasionally affect salinity intrusion by diluting salinity and transporting it out of Lake Nakaumi. It also affects sea surface elevation in Sakai Channel and promoting salinity intrusion through the bottom layer. Wind drift has lesser effect but occasional froing winds promote circulation and either enhance or inhibits salinity intrusion.

River discharge is the only forcing parameter that may be controlled through damming upstream. Increased river discharge has the capacity to flush salinity of the lakes, however it also promotes salinity intrusion through the bottom according to previous studies (Nakata et al. 2000). Management of water flow in the Hii River system may be important in the mitigation of the impact of seawater intrusion to lakes ecosystems. To understand the effect of various river discharge scenarios on salinity transport, further studies using numerical models are required. Ministry of Land, Infrastructure, Transport and Tourism (MLIT) is promoting a flood management project, which constitutes construction of new reservoirs in the upper reaches of the Hii River and in the Kando River (Seigel 2012). The project will also construct a diverging channel connecting the Hii River and the Kando River, in conjunction with widening and deepening of Ohashi River. Since the study indicated that river discharge plays an important role in the saltwater intrusion process, the impact of these projects on salinity intrusion processes need to be studied. The widening of Ohashi River has impacts on the flow rate per cross-sectional area which may promote/inhibit seawater intrusion. Hence these projects should be included in models to come up with river system management practices for effective seawater intrusion management. The study attempted to analyse the mechanisms that drove salinity transport through Nakaura Watergate before it was removed. New studies need to be carried out to understand the effect of the removal of the flood gates.

\section{Conclusion}

Salinity intrusion in Lakes Shinji and Nakaumi controls their salinity level and thus affects their ecosystems. In order to analyse the time series of salinity flux, $M_{s}$, at the Nakaura Watergate in the Sakai Channel and to reveal how forcing parameters affect its variation, recent statistical methodology of the continuous wavelet transform, CWT, and the partial wavelet coherence, PWC, were utilized. Based on the previous researches, the explanatory parameters selected were tidal level, river discharge, atmospheric pressure, and the wind vector. The study results reveal the fundamental characteristics in variation of such forcing parameters and salinity flux themselves, as well as their interactions.

The continuous wavelet transform results show that salinity flux mainly has 12 -h and 1-day periods. Tide level shows periodical variation around 0.5 and 1-day period, indicating the dominants of the astronomical tides. Hii River discharge does not show any periodical characteristics because of unpredictability of precipitation and controlled release from upstream reservoirs. Atmospheric pressure exhibits continuous high power with an average 6-day period affecting sea surface elevation occasionally. Wind data shows stable oscillations with periods between 8 and 32-day period.

Partial wavelet coherence and partial phase difference revealed the influence of tide level and river discharge on salinity flux. Although the results suggest that atmospheric pressure and north-south wind vector have little influence on the salinity flux at Nakaura Watergate, it is possible for them to indirectly influence salinity transport through their effect on sea surface elevation. High pressure drops occasionally resulted in increased tide level. The geometry of Sakai channel may limit wind to cause changes only in the surface currents, hence weak effect on the overall transport of salinity. The study quantified the phase lags between salinity flux and tide level; the peak of tide level was an average of 4 ahead of salinity flux and low water was 2.3 days ahead. Almost half the time, tide level was leading salinity flux. River discharge was about 0.3 days leading salinity flux. Almost $40 \%$ of the time, river discharge was leading salinity flux. This study found that salinity transport in the Sakai Channel increases with the increase of river discharge upstream. Further studies are needed to understand different river discharge regimes and their impacts on salinity intrusion, and the effect of the removal of Nakaura Watergate, taking into consideration the proposed MLIT flood management project.

Acknowledgements Shimane Prefectural Institute of Public Health and Environmental Science provided the salinity, water temperature and current velocity data used in the study. The two anonymous reviewers provided valuable comments. Wavelet packages used in the study were developed by L. Aguiar-Conraria, and M. J. Soares (http:// sites.google.com/site/aguiarconraria/joanasoares-wavelets); $\mathrm{Ng}, \mathrm{E}$. K. W. and J. C. L. Chan, (http://www.cityu.edu.hk/gcacic/wavelet/); and A. Grinsted, J.C. Moore and S. Jevrejeva (http://www.pol.ac.uk/ home/research/waveletcoherence).

Open Access This article is distributed under the terms of the Creative Commons Attribution 4.0 International License (http://crea tivecommons.org/licenses/by/4.0/), which permits unrestricted use, distribution, and reproduction in any medium, provided you give appropriate credit to the original author(s) and the source, provide a link to the Creative Commons license, and indicate if changes were made.

\section{References}

Aguiar-Conraria L, Soares MJ (2014) The continuous wavelet transform: moving beyond uni- and bivariate analysis. J Econ Surv 28(2):344-375 
Briciu A-E (2014) Wavelet analysis of lunar semidiurnal tidal influence on selected inland rivers across the globe. Sci Rep 4:4193

Fujii T, Okuda S (2004) The Relationship between internal waves and the movement of anoxic water in two connected brackish water bodies-Lake Nakaumi and the Ohashi River. In International Seminar on Restoration of Damaged Lagoon Environments. Japan: Matsue, p 1.

Grinsted A, Moore JC, Jevrejeva S (2004) Application of the cross wavelet transform and wavelet coherence to geophysical time series. Nonlinear Process Geophys 11(5/6):561-566

Harada E (1968) Ecology and biological production of Lake Nakaumi and adjacent regions -5 . Seasonal changes in distribution and abundance of some decapod crustaceans. Special Publications from the Seto Marine Biological Laboratory, 2(2) pp 75-103

Ideião SMA, Santos CAG (2005) Analysis of precipitation time series using the wavelet transform. Sociedade \& Natureza, Uberlandia, (Special Issue), 736-745

Ishitobi Y et al (1989) The tidal exchange in Lake Shinji under low discharge conditions. Jpn J Limnol (Rikusuigaku Zasshi) 50(2): 105-113

Ishitobi Y et al (1999) Physical conditions of saline water intrusion into a Coastal Lagoon, Lake Shinji, Japan. Jpn J Limnol (Rikusuigaku Zasshi) 60(4):439-452

Klinger BA Density of Seawater, p 4. http://mason.gmu.edu/ $\sim$ bklinger/seawater.pdf

Liu B et al (2014) Wavelet analysis of the dynamic characteristics of saltwater intrusion-A case study in the Pearl River Estuary of China. Ocean Coast Manag 95:81-92

Mizoyama I, Ohya Y, Fukuoka S (2011) Flow mechanism in a connected Brackish Lake and numerical flow computation over a long period. Journal of Japan Society of Civil Engineers, Ser. B1 (Hydraulic Engineering), 67(3): 101-120

Moser GAO et al (2005) Instantaneous Transport of Salt, Nutrients, Suspended Matter and Chlorophyll-a in the Tropical Estuarine System of Santos. Braz J Oceanogr 53(3/4):115-127

Nakata K, Horiguchi F, Yamamuro M (2000) Model study of Lakes Shinji and Nakaumi-a coupled coastal lagoon system. J Mar Syst 26(2):145-169
Ng EKW, Chan JCL (2012) Geophysical applications of partial wavelet coherence and multiple wavelet coherence. J Atmos Ocean Technol 29(12):1845-1853

Okuda S (2004) Water movement and physical environments in brackish lakes-Shinjiko and Nakaumi. In International Seminar on Restoration of Damaged Lagoon Environments. Matsue, Japan, 1-6.

Parmar KS, Bhardwaj R (2013) Wavelet and statistical analysis of river water quality parameters. Appl Math Comput 219(20): 10172-10182

Seigel MT (2012) Consensus building revisited: the experience and Approach of Toshio Kuwako. Centre for Dialogue Working Paper Series, p 54

Somoza RD et al (2013) A water level relationship between consecutive gauge stations along Solimoes/Amazonas main channel: a wavelet approach. arXiv: 1310.8527

Sousa RMDD (2014) Carbon prices. Dynamic analysis of European and Californian markets. Doctoral Dissertation. Universidade Nova de Lisboa, Maio

Sovi A et al (2012) Wavelet analysis of hydrological signals on an example of the River Sava. In: MIPRO, 2012 Proceedings of the 35 th international convention. IEEE, Opatija, pp. 1223-1228

Torrence C, Compo GP (1998) A practical guide to wavelet analysis. Bull Am Meteorol Soc 79(1):61-78

Uye S et al (2000) Geographical and seasonal variations in mesozooplankton abundance and biomass in relation to environmental parameters in Lake Shinji-Ohashi River-Lake Nakaumi brackish-water system, Japan. J Mar Syst 26(2):193207

Vaz N, Lencart e Silva JD, Dias JM (2012) Salt Fluxes in a Complex River Mouth System of Portugal J. Kurths, ed. PLoS ONE 7(10):e47349

Yamamuro M, Nakamura M, Nishimura M (1990) A method for detecting and identifying the lethal environmental factor on a dominant macrobenthos and its application to Lake Shinji, Japan. Mar Biol 107(3):479-483

Zhang Q, Xu C-Y, Chen YD (2009) Wavelet-based characterization of water level behaviors in the Pearl River estuary, China. Stoch Env Res Risk Assess 24(1):81-92 